\title{
Design of Remote Grain and Oil Data Updating Based on .NET Platform Lihua Jiang ${ }^{1, a^{*}}$ and Chujun Zhang ${ }^{2, b}$

\author{
${ }^{1}$ School of Computer, Wuhan Polytechnic University, Wuhan Hubei, 430023, China \\ ${ }^{2}$ School of Computer, Wuhan Polytechnic University, Wuhan Hubei, 430023,China \\ ajianglihua@whpu.edu.cn, b498761101@qq.com
}

\section{Keywords: ASP.NET; Stored Procedure; Data update}

\begin{abstract}
From a simple and practical point of view, a method of updating remote grain and oil data by stored procedure is proposed. This paper mainly explains how to establish the stored procedure in SQL Server and problems which were encountered when data updating was being implemented in ASP.NET, besides, also gives the key program of the method to realize the code analysis. The experience shows that the method adopting stored procedure to manipulate data not only improves the speed of data access, but also shields the logic of database table well. It has a good reference value for the development of whatever management information system with three-layer B/S structure.
\end{abstract}

\section{Introduction}

The promotion of national grain depot's informatization not only is conducive to the integrity and standardization of the grain enterprises' business process, but also is beneficial for the automation of grain data collection. However, most of the grain depots' work mode is obviously backward. Its working efficiency is low, furthermore, that the artificial collection of grain information is inaccurate and the accounts are not in conformity with the reality are the common phenomena. In this context, the author used the .NET2003 Microsoft recently launched as the development platform, the efficient and quick ASP.NET as the development tool, and the safe and reliable SQL Server2000 as a backstage database to design and develop Hubei Shekou grain depot management information system, which realized the organic combination and integrated management between grain capital management and logistics management, avoided the mismanagement resulting from the nonstandard operation, reduced the daily work intensity and enhanced the management quality and level of the grain system. In this paper, the method of using stored procedure to update the remote database data in ASP.NET is introduced, which is simple and reliable, and has been successfully applied to the "grain depot management system based on B/S model " developed by the author.

\section{Configuration of the Database for the Management Information System}

Database Preparation. Take the SQL Server2000 database as an example. First, set up a database named userdb, and then establish a grain price specified table named jiagezhiding with 5 fields in the database.The fields are as follows: the structure ID field is i_jiageid, int, self growth; the name field is c_pinming, varchar (50); the moisture field is d_shuifen, numeric $(18,2)$; the price field is $\mathrm{d}$ jiage, numeric (18 2); the memo field is c_memo, varchar (100). The memo field is mainly used to either record some useful information or expand the system.

In view of the security of the database, establish a user named guest (the password is also guest), and then add the login name guest to the users list of the database userdb, subsequently grant the select, insert, delete, update permissions of the table jiagezhiding for the user userdb. User guest is only used to connect to the database and get the user's password.

Implementation of Stored Procedure. For developers using ASP.NET to develop B/S pattern management information systems, it is a key and complex problem to ensure the efficiency of database data processing. The grain depot management system involves multiple data tables and stored procedures. In view of the limited space, only one table and one stored procedure are used as 
an example to complete remote data update. In fact, when the vast majority of the network applications based on WEB access database data, not directly contact with database tables through the SQL statements, but rely on the operation of stored procedures to obtain the required data. The advantage of this designment is to avoid frequent table operations, and to perform corresponding operations after running the stored procedure to obtain parameters in the server side, which can greatly improve the operating efficiency and the speed of accessing data, while shielding the logic of the database table. This makes that the database access becomes the service access provided by the database.

The data manipulations of the whole grain depot management system depend on stored procedure. For every table in the database, corresponding to 4 stored procedures at least, namely, query, add, delete and modify the required record. Stored procedures use the input parameters to get the corresponding information from the client, and the operation of the database tables is completed when the stored procedure is executed.

The stored procedure update jiagezhiding used to update the data of the table jiagezhiding is defined as follows:

CREATE PROCEDURE [update_jiagezhiding]

(@i_jiageid_1_[int],

ac_pinming_2 [varchar] (50),

ad_shuifen_3 [numeric],

(a) jiage $4^{-}$[numeric])

AS UPDATE [userdb].[dbo].[jiagezhiding]

SET [c_pinming] =@c_pinming_2,

[d_shuifen] =@d_shuifen_3,

[d_jiage] $=@$ d jiage_4

WHERE ( [i jiageid] $=\bar{@}$ i jiageid_1)

Using the same method, the stored procedures of select, insert, and delete can be established, and the corresponding stored procedures can be invoked and executed only by passing parameters in the application. There is an example to illustrate that using stored procedure to update database data.

\section{Using Stored Procedures to Complete the Data Update}

Specifying the Database Connection String. First, start Visual Studio.NET, then create a ASP.NET Web application named "LiangKuApp" in the Visual Basic project option group. In order to maintain the good portability of the system, the method of uniformly managing the database is adopted, namely, all the information used in the program to connect database string is uniformly placed in the web.config configuration file or the global.asax global file. These string configuration information is called in the program, in order to realize the unified revision and usage.

Set up the Connectimg Database String in the Web.config Configuration File. The web.config configuration file is a document based on XML, which can set up the connecting database string which can be implement by applying the appsettings tag under the configuration tag. The code is as follows:

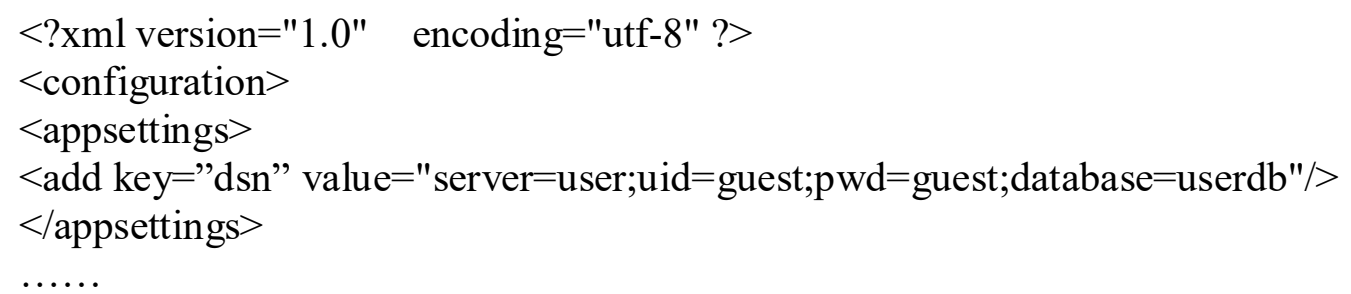

The database settings information of the configuration document in the program is as follows:

Dim strconn As String = ConfigurationSettings.AppSettings("dsn")

Use the Session object to Set up the Connecting database String in the Global.asax Global File. Use the session object of the server to record the connecting database string. The session variable acts on each session, and the following code is added to the global.asax global file:

Sub Session_Start(ByVal sender As Object, ByVal e As EventArgs) 
End Sub

' Excited when the session starts.

Session("strpub") = "server=user;uid= guest;pwd= guest;database=userdb"

The following details are applied to introduce how to use the ADO.NET to access database and use the update_jiagezhiding stored procedure to update the data in the data table jiagezhiding in the grain depot management system.

Implementation of Data Update. The .NET framework of Microsoft provides convenience for network programming. It is the emergence of ASP.NET that makes the development of the application based on WEB services simple and efficient. Especially for the processing of the database, it is much more convenient than the original ASP. The following example is the implementation of jiagezhiding data table updating by using ADO data access method.

Rename the webforml.aspx of the "LiangKuApp" ASP.NET Web application to jiagezhiding.aspx, and insert 4 Label controls, 3 Textbox controls, and 8 Button controls on the jiagezhiding.aspx window. As shown in Fig. 1, the ID properties of the 3 text boxes after name, water, and price tags are Textbox1, Textbox2, and Textbox3.

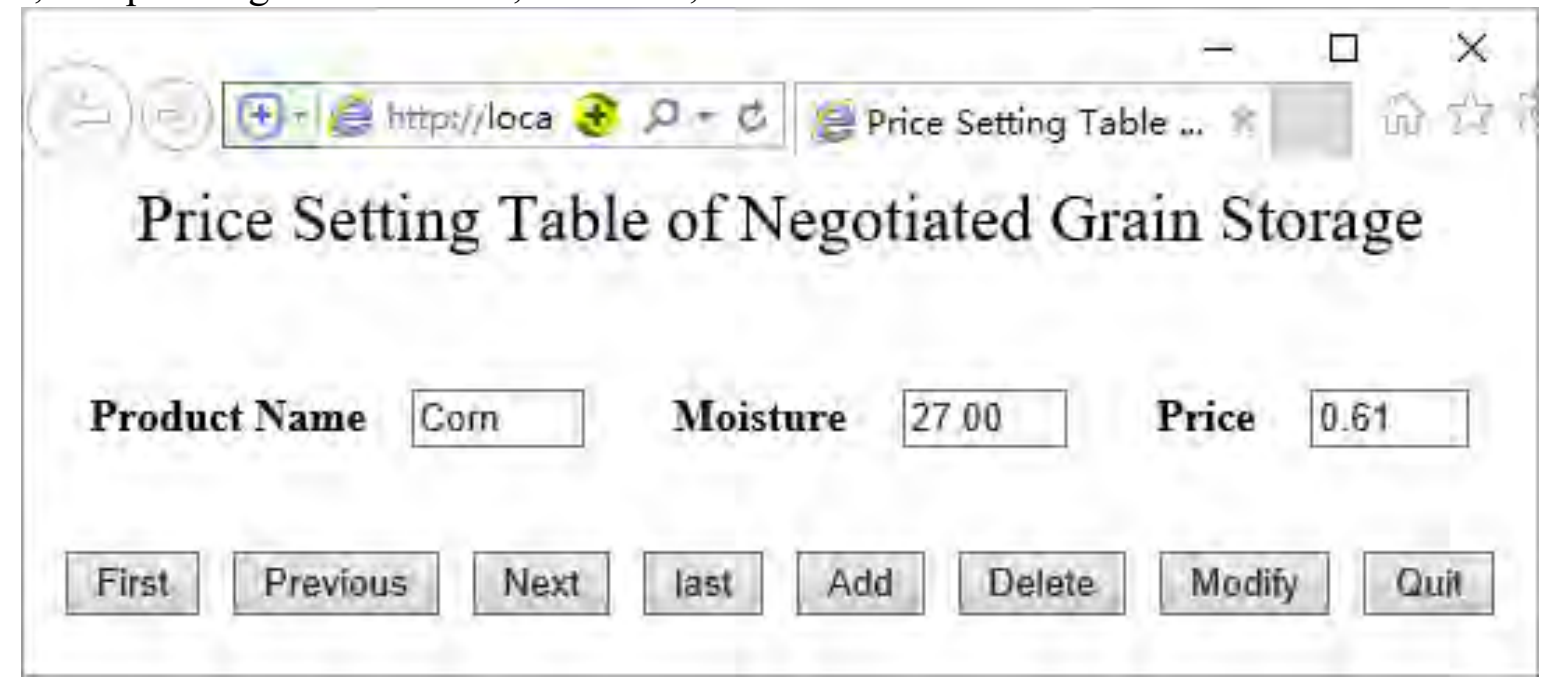

Figure 1. The price setting page

Then double click the jiagezhiding.aspx to view the code window, that is, add the Imports statement above the jiagezhiding.aspx.vb's class declaration for importing the namespace required to access relational data. The following Imports statements can be used in this instance:

Imports System.Data

Imports System.Data.Sq1Client

The Click event is created for modification (update) button Button7. The code is as follows:

Private Sub Button7_Click(ByVal sender As System.Object, ByVal e As System.EventArgs) Handles Button1.Click

Dim cn As New SqlConnection(Session("strpub"))

' Using Session ("strpub") variable to read the connecting string to connect database.

cn.Open() 'Open the database.

Dim cm As New SqlCommand("update jiagezhiding", cn)

' Using the object SqlCommandto call the stored procedure update jiagezhiding. $\mathrm{cm}$.CommandType $=$ CommandType.StoredProcedure $\quad$ 'Turn the command type into a storage type.

cm.Parameters.Clear() 'Parameters scavenging. cm.Parameters.Add("@i_jiageid_1", Sq1DbType.int) ' Add parameters and assign values. cm.Parameters.Add("@c pinming_2", SqlDbType.NVarChar)

cm.Parameters.Add("@d_shuifen_3", SqlDbType.NVarChar)

cm.Parameters.Add("@d_jiage_4", SqlDbType. Decimal)

cm.Parameters("@c_pinming_2").Value=Trim(TextBox1.Text) 
cm.Parameters("@d_shuifen_3").Value=Trim(TextBox2.Text)

cm.Parameters("@d_jiage_4").Value=Trim(TextBox3.Text)

cm.Parameters("@i_jiageid_1").Value=session(jiageid)

' session(jiageid) is the value of the $\mathrm{i}$ jiageid field currently recorded in the program.

cm.ExecuteNonQuery() ' Execute stored procedure without returning result. Data table

jiagezhiding data is updated.

cn.Close() ' Close the database connection.

Response.Redirect("User modified successfully! ") ' Output the prompt message about that information has been modified successfully.

End Sub

After the completion of the operation, compile it, and you can type the server's address to handle the data in the client's browser, such as http://211.85.205.169/ LiangKuApp/jiagezhiding.aspx. When the application runS to the page as shown in Figure 1, the administrator clicks the corresponding button to access the specified record. If you want to change the data, you can modify data in the text box on this page. And then click "modify" button to complete data update in data table jiagezhiding.

For the completion of add and delete functions, we can also refer to the above methods. Specify different stored procedures to transfer corresponding parameters, and complete corresponding functions. The grain depot management system is debugged on ASP.NET2010 and SQL Server2015 platform. All stored procedures have good effect.

\section{Conclusions}

The management system of the grain depot is based on the.NET2003 platform of Microsoft. The above code has been successfully applied in the system, and it is very simple to realize, reliable in operation. Due to using a standard SQL statement, it can also be applied to other large databases. It fully uses and embodies the Web Services mechanism, and establishes a simple and efficient three-layer B/S structure application system. The system composed of business appearance layer, business logic layer and data access layer has high safety and reliability, also provides a model for national grain depot construction.

At present, the management system of the grain depot has been applied to Hubei Shekou grain depot. By adopting this system, the schedule and quality of the farmers' grain settlement are remarkably improved, the cost of the purchase and sale enterprises is saved, the supervision of the stock is strengthened, and the closed operation management is on a new step.

\section{References}

[1] Sun Yong-qiang. Visual C\#.NET Chinese Version Web Service Development Foundation [M]. Beijing: Tsinghua University Press, 2014.8

[2] Xue Wei-min, Xie Lun. Visual Basic.NET Web Application Development. Beijing : Tsinghua University Press, 2015.4

[3] John Kauffman, Chris Goode, Brian Matsik. Application Architecture for .NET: Designing Applications and Services, www. microsoft. com, 2015.12.24

[4] Four Dimensions Technology, Cao Yan-long. Selection of Practical Engineering Cases for ASP/ASP.NET Database Development. Beijing: The Posts and Telecommunications Press, 2012.7

[5] GUO Xiaofeng,YAO Shijun,YIN Zuwei.Design and appoication of framework of web application based on.NET[J].Computer Engineering and Design,2011,29(2):424-428.

[6] CHEN Qi,ZHANG Lianhai,CAO Yemin.Design and imple-mentation of .NET-based metering information management system[J].Computer Engineering and Design,2011,28(6):820-822.

[7] WANG Chaoyang.Web application system security technology based on .NET[J].Network Security Technology \& Application,2012(10):92-94. 
[8] Matthew Mac Donald. ASP.NET Advanced Programming[M]. Beijing:Posts and Telecommunications Press, 2011.

[9] DAVID S.Microsofe ADO.Net:Core Reference[M].Microsoft Press Redmond,WA,USA 2009.

[10]PAN Jie,ZHOU Chuansheng. Web Research and Implementation Based on $\mathrm{j}$ Query Framework[J].Journal of Shenyang Normal University:Natural Science,2014,32(1):94-97. 